\title{
The Impact of Cooperative Adaptive Cruise Control on Traffic-Flow Characteristics
}

\author{
Bart van Arem, Member, IEEE, Cornelie J. G. van Driel, and Ruben Visser
}

\begin{abstract}
Cooperative adaptive cruise control (CACC) is an extension of $\mathrm{ACC}$. In addition to measuring the distance to a predecessor, a vehicle can also exchange information with a predecessor by wireless communication. This enables a vehicle to follow its predecessor at a closer distance under tighter control. This paper focuses on the impact of CACC on traffic-flow characteristics. It uses the traffic-flow simulation model MIXIC that was specially designed to study the impact of intelligent vehicles on traffic flow. The authors study the impacts of CACC for a highway-merging scenario from four to three lanes. The results show an improvement of traffic-flow stability and a slight increase in traffic-flow efficiency compared with the merging scenario without equipped vehicles.
\end{abstract}

Index Terms-Adaptive cruise control (ACC), intelligent vehicles, traffic-flow simulation, vehicle-vehicle communication.

\section{INTRODUCTION}

D URING THE past few decades, western society has been constantly confronted with problems caused by increasing road traffic. This increase in traffic demand leads to a heavily congested network and has a negative effect on traffic safety, air pollution, and energy consumption. The expectations of the use of telematics technology in road traffic in this respect are high, since this technology could lead to system innovations (e.g., advanced vehicle guidance), which in the long term can contribute to the problems faced [1], [2].

Advanced driver-assistance (ADA) systems support a driver in his driving tasks. These systems are being developed because they have the perspective to increase the driver's safety and comfort. Additionally, ADA systems can have a positive impact on traffic-flow performance and reduce emissions and fuel consumption. Examples of ADA systems are various forms of cruise control, lane-keeping systems, and collision-warning systems.

Manuscript received October 14, 2005; revised June 20, 2006, July 31, 2006, and August 4, 2006. This work was supported by the Applications of Integrated Driver Assistance (AIDA) program of The Netherlands Organization of Applied Scientific Research TNO and the University of Twente. The Associate Editor for this paper was L. Vlacic.

B. van Arem and C. J. G. van Driel are with the Research Program of Applications of Integrated Driver Assistance (AIDA), Centre for Transport Studies, Faculty of Engineering Technology, University of Twente, 7500 Enschede, The Netherlands (e-mail: b.vanarem@utwente.nl; c.j.g.vandriel@ utwente.nl.).

R. Visser was with the Research Program of Applications Integrated Driver Assistance (AIDA), Centre for Transport Studies, Faculty of Engineering Technology, University of Twente, 7500 Enschede, The Netherlands. He is now with 4Motion Consultancy, 3521 Utrecht, The Netherlands (e-mail: ruben@ 4motion.nl).

Digital Object Identifier 10.1109/TITS.2006.884615
An ADA system that has been introduced by the automotive industry is adaptive cruise control (ACC). ACC is a radar-based system, which is designed to enhance driving comfort and convenience by relieving the driver of the need to continually adjust his speed to match that of a preceding vehicle. The system slows down when it approaches a vehicle with a lower speed, and the system increases the speed to the level of speed previously set when the vehicle upfront accelerates or disappears (e.g., by changing lanes).

Vehicle-to-vehicle communication can further advance the development of ADA systems. Cooperative ACC (CACC) is a further development of ACC that adds vehicle-to-vehicle communication, providing the ACC system with more and better information about the vehicle it is following. With information of this type, the ACC controller will be able to better anticipate problems, enabling it to be safer, smoother, and more "natural" in response. Although CACC is primarily designed for giving the driver more comfort and convenience, CACC has a potential effect on traffic safety and traffic efficiency. It is of importance to understand the traffic-flow effects of CACC early in the development so that, if they are discovered to inadvertently create problems, the design can be adjusted accordingly before adverse traffic effects are widely manifested. Apart from that, it is recommended to study the traffic-flow effects of CACC so that these (comfort) systems can be developed to best support future advances.

Uncertainties exist about the traffic-flow impact of the relatively new developed system CACC. The objective of this paper is to assess the impact of CACC on traffic-flow characteristics in terms of traffic stability and throughput.

This paper is organized as follows. In Section II, we review the relevant literature. In Section III, we describe the trafficflow simulation model MIXIC. The CACC system is then explained in Section IV. We describe the setup of the simulation study and its results in Sections V and VI, respectively. The results are discussed in Section VII. Section VIII contains our conclusions.

\section{REVIEW OF LITERATURE}

According to two literature studies, ACC can contribute to the stability of traffic flow limitedly and affect traffic performance both positively and negatively [3], [4]. A lowpenetration level of ACC does not have any effect on traffic flow, regardless of the time-gap set [5]. Even under the most favorable conditions, with ideal ACC system design and performance, it appears that ACC can only have a small impact on highway capacity [6]. 
In contrast to the voluminous literature on autonomous ACC, the literature related to CACC is limited. In a number of studies, the functionality, architecture, or design of CACC systems have been described. However, extensively exploring the traffic-flow effects, quantitatively in terms of throughput and capacity, has been done by only a few researchers.

The concept of full platooning is described in [7]. Highcapacity values of up to 8500 vehicles an hour per lane can be achieved, if separate infrastructure is available and all vehicles using this lane can communicate with each other. This concept of automated highway systems (AHSs) is especially envisioned by American researchers. AHS have been defined as vehicle-highway systems that support hands-off and feet-off driving on dedicated freeway lanes. Different AHS have been explored, and some have been investigated in depth.

Cooperative following (CF) uses automated longitudinal control combined with intervehicle communication [8]. It allows for anticipation to severe braking maneuvers in emerging shock waves with the aim of smoothening traffic flow and enhancing traffic safety. The functionality of $\mathrm{CF}$ has been modeled in the microscopic-traffic simulation model MIXIC, and the simulation has been run with a platoon of mixed $\mathrm{CF}$ equipped and nonequipped vehicles. Although at a platoon level better stability was achieved, the potential advantages on trafficflow efficiency could not be confirmed.

The CarTALK 2000 project focuses on developing cooperative driver-assistance systems, which are based upon mobileintervehicle communication [9]. The traffic impacts of two applications [basic warning function and early braking (i.e., a continuation of $\mathrm{CF}$ )] were assessed using MIXIC. Both applications concern vehicles that broadcast a message to other vehicles when accelerating with $-2.0 \mathrm{~m} / \mathrm{s}^{2}$ or less. The results indicate an improvement of traffic stability in terms of a reduction in the number of shockwaves for all penetration rates and headways tested.

The effects on capacity of increasing market penetration of both ACC and CACC vehicles, relative to manually driven vehicles, was examined in a quantitative way by using microscopic-traffic simulation [6]. A single highway lane with a ramp-highway junction consisting of a single-lane offramp followed immediately by a single-lane onramp has been considered. The analyses were initially conducted for the distinct cases of $100 \%$ manually driven vehicles, $100 \%$ ACC (time gap of $1.4 \mathrm{~s}$ ), and $100 \%$ CACC (time gap of $0.5 \mathrm{~s}$ ) to verify the reasonableness of the results under these simplest cases. The nominal-capacity estimates for the manual driving, ACC, and CACC cases were 2050, 2200, and 4550 vehicles per hour, respectively. Next, mixed vehicle populations were analyzed in all feasible multiples of $20 \%$ of each vehicle type. It was concluded that CACC can potentially double the capacity of a highway lane at a high-CACC market penetration. The capacity effect is sensitive to market penetration, based on the fact that the reduced time gaps are only achievable between pairs of vehicles that are CACC equipped.

The CHAUFFEUR 2 project has addressed three approaches aiming to reduce a truck driver's workload by developing truckplatooning capability [10]. First, we have an electronic tow bar in which a vehicle automatically follows a manually driven leading vehicle. Due to vehicle-to-vehicle communication, the following distance is very close $(6-12 \mathrm{~m}$, which is equal to $0.3-0.6 \mathrm{~s}$ at $80 \mathrm{~km} / \mathrm{h})$. Second, we have the Chauffeur assistant, which enables the truck to follow any other vehicle on a highway with a safe following distance using an ACC and a lane-keeping system. Third, we have electronic coupling of three trucks in a platooning mode. The leading vehicle is driven conventionally, and both following vehicles follow. Also, in this project, the following distance is very close $(6-12 \mathrm{~m})$. For this last platooning mode, the trucks are equipped with vehicleto-vehicle-communication systems. Considering the results of a simulation study with the microscopic-traffic simulation models VISSIM and FARSI, it is concluded that the main effects of the CHAUFFEUR 2 systems are a better usage of road capacity, up to $20 \%$ reduction in fuel consumption, and increased traffic safety. However, it has been remarked that platooning is mostly feasible at night or on sections with low-traffic volume because, during high-traffic volume, the stability of traffic flow decreased.

From this literature review, the following conclusions can be drawn. First, vehicle-to-vehicle communication can provide an ACC system with more and better information about the vehicle it is following. Not only following distance and speed difference with respect to its direct predecessor are considered, but also speed changes can be coordinated with each other. The information could include precise speed information, acceleration, fault warnings, warnings of forward hazards, maximum braking capability, and current braking capability. With information of this type, the ACC controller can better anticipate problems, enabling the vehicle to be safer, smoother, and faster in response and, as a result, enable closer vehicle following. Time gaps could be as small as $0.5 \mathrm{~s}$.

Second, CACC has the potential to increase capacity by minimizing time gaps between consecutive vehicles and trafficflow stability by improving string stability. This improved performance can only be achieved when pairs of vehicles are equipped with the CACC system. Therefore, the improvement of traffic efficiency largely depends on the level of CACC deployment.

Third, extensive research into the traffic-flow impact of CACC, in terms of traffic-flow stability and throughput, is lacking. The limited CACC effect studies that have been performed emphasize that $\mathrm{CACC}$ is able to increase the capacity of a highway significantly. CACC can potentially double the highway capacity at a high market penetration.

\section{TRAFFic Simulation Model MIXIC}

In order to study the potential impact of ADA systems, such as CACC, the modeling approach should be suitable for analyzing different assumptions for ADA-system functionality, roadside systems, driver behavior, and vehicle dynamics. Furthermore, it should be capable of assessing impacts on traffic performance, traffic safety, exhaust-gas emission, and noise emission. In order to meet these requirements, a stochastic simulation model MIXIC was developed [11].

The microscopic-traffic simulation model MIXIC simulates traffic on a link level in a network. Given an input of traffic flow, 
MIXIC simulates traffic behavior on this link and produces traffic statistics. MIXIC uses real traffic measurements (time instant, lane, speed, and vehicle length) to generate traffic at the start of the simulation run. At each step (set to $0.1 \mathrm{~s}$ ), new vehicle positions are calculated by a driver model and a vehicle model. The driver model produces driver actions, such as lane changing and new pedal and gear positions. These driver actions are input for the vehicle model, which calculates the resulting acceleration and position of the vehicle. The main components of MIXIC are described in the remainder of this section.

The traffic generator decides whether or not to place new vehicles at the start of the first road link. Vehicles are generated from so-called traffic "injection" files and are assigned specific vehicle/driver data and an initial state. A traffic injection file consists of recorded real-world data of individual vehicles (arrival time, position, lane, speed, and length). Vehicle types and driver types (reaction time, desired speed, etc.) are assigned randomly (using occurrence frequencies). The use of injection files to generate input for a microscopic model has three advantages. First, it is by definition realistic. Second, it puts the traffic-evolution model to the test (this model should in any case be able to process the amount of traffic offered by the injection file, traffic being actually observed). Third, it allows for calibration and validation by comparing the model with measurement further downstream [12].

The driver model consists of three main components: the lane change model, the longitudinal model, and a component, which describes the interaction between the driver and the ADA system. The lane-change model consists of a mandatory lane-change model (represents forced lane changing due to geometric factors) and a free lane-change model (represents overtaking). The longitudinal model distinguishes free-driving behavior (the driver attempts to reach or maintain his intended speed) and car-following behavior (the driver adjusts his speed and/or following distance with respect to traffic ahead). The carfollowing model implemented in MIXIC is derived from the optimal control model of Burnham et al. [13]. It is based upon the assumption that drivers try to keep the relative speed to the lead car zero and simultaneously attempt to keep the clearance at a desired value. In addition to the original model, also, the relative speed to the vehicle ahead of the lead vehicle is taken into account because it contributes to the stability of the traffic flow [12]. In contrast with other microscopic-traffic models, it is possible in MIXIC to specify the driver interaction with an ADA system.

The vehicle model describes the dynamic vehicle behavior as a result of the interaction with the driver and the road, taking into account the ambient conditions. The vehicle model uses information on the characteristics of the vehicle, the road geometry, the condition of the road, and the wind. The output of the model is an updated vehicle acceleration, which is used to calculate a new speed and position of the vehicle.

To study the effects of ADA systems, MIXIC also contains a detailed model describing the behavior of the ADA system in the paper. An ACC model is already available in MIXIC. If the driver has switched on the ACC system, the ACC model takes over the longitudinal driver model. To obtain the information

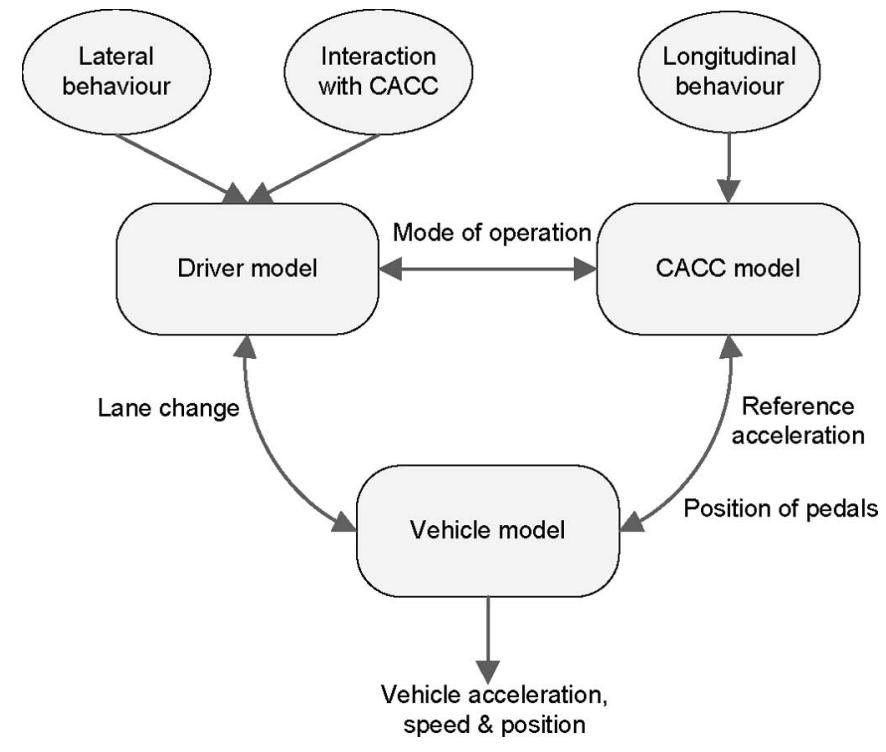

Fig. 1. Position of CACC model in MIXIC.

needed for this control task, a radarlike sensor is used. The sensor is characterized by its delay and its maximum detection range. Failures in the operation of the sensing and communication equipment were not considered.

The simulation model MIXIC was originally developed in the early 1990s. It was calibrated for different two-, three-, and four-lane motorway situations. The motorway situation studied in this paper was calibrated against data from the A4 motorway near Schiphol [12], [14]. Both the longitudinal and lateral models appeared to represent real-life traffic adequately. It appeared that the traffic flows on a four-lane setting were considered realistic. On the part where the traffic merges to a three-lane motorway, traffic volumes as high as $7700 \mathrm{pcu} / \mathrm{h}$ were observed for 5-min intervals. Although this appeared to be high, empirical studies have indicated that such peak flows are indeed high but not unrealistic [15].

\section{Cooperative Adaptive Cruise Control (CACC)}

The characteristics of MIXIC are suitable for exploring the impact of CACC on the traffic flow. First, however, a new CACC model had to be designed in MIXIC. The basis of the longitudinal driver model in MIXIC is the calculation of a desired acceleration of a driver. If a CACC system takes over a part of the longitudinal driving task of a driver, a reference acceleration of the CACC controller is calculated instead. This reference acceleration is used to determine the real acceleration of the vehicle in the MIXIC vehicle model. The position of the CACC model in MIXIC as described above is presented in Fig. 1.

For modeling purposes, a CACC-equipped vehicle can be divided into two distinct components: the CACC controller delivering reference values and a vehicle model transforming the reference values into actually realized values, using the vehicle model. The acceleration reference demand from the CACC controller must be determined and then fed into the vehicle model. The equations for the speed and distance controller have been derived from [16]. Since MIXIC differs from the model 
used in [16], some changes have been made in these algorithms in order to develop a workable model.

The acceleration demand can be computed on the basis of the difference between current and intended speed $\left(a_{\text {ref_}} \nu\right)$ or on the basis of the distance and speed difference between the ego vehicle (i.e., CACC-equipped vehicle) and target vehicle (i.e., predecessor of ego vehicle) $\left(a_{\text {ref_d }}\right)$. The acceleration demand is given by the most restrictive one:

$$
a_{\text {ref }}=\min \left(a_{\text {ref_} \_}, a_{\text {ref_d } d}\right) \text {. }
$$

The resulting reference acceleration is limited between the maximum comfortable acceleration $2 \mathrm{~m} / \mathrm{s}^{2}$ and the maximum comfortable deceleration $-3 \mathrm{~m} / \mathrm{s}^{2}$.

Let $\nu_{\text {int }}$ and $\nu$ denote the intended and current speed, respectively, of the CACC set by the driver in meters per second. The acceleration demand, based on speed difference, is given by

$$
a_{\text {ref } \_\nu}=k \cdot\left(\nu_{\text {int }}-\nu\right)
$$

with $k$ as a constant-speed error factor.

The computation of the reference acceleration, based on the distance and speed difference between the ego vehicle and target vehicle, is slightly more complicated. Let $\nu_{p}$ denote the speed of the target vehicle, and let $r$ and $r_{\text {ref }}$ denote the current and reference clearance to the target vehicle in $m$, respectively. Let $a_{p}$ denote the acceleration of the target vehicle. The reference acceleration, based on the distance and speed difference between the ego vehicle and target vehicle, is given by

$$
a_{\text {ref_d }}=k_{a} \cdot a_{p}+k_{\nu} \cdot\left(\nu_{p}-\nu\right)+k_{d} \cdot\left(r-r_{\text {ref }}\right)
$$

with $k_{a}, k_{\nu}$, and $k_{d}$ as constant factors.

The reference clearance $r_{\text {ref }}$ is defined as a maximum among safe following distance $\left(r_{\text {safe }}\right)$, following distance according to the system time setting $\left(r_{\text {system }}\right)$, and a minimum allowed distance $\left(r_{\min }\right)$, set at $2 \mathrm{~m}$. The safe following distance is computed on the basis of the speed $\nu$ of the ego vehicle and the deceleration capabilities $d$ and $d_{p}$ of the ego vehicle and the target vehicle, respectively:

$$
r_{\mathrm{safe}}=\frac{\nu^{2}}{2} \cdot\left(\frac{1}{d_{p}}-\frac{1}{d}\right)
$$

where, for simplicity, we have assumed a communication delay equal to zero. The following distance according to the systemtarget time-gap setting is given by

$$
r_{\mathrm{system}}=t_{\mathrm{system}} \cdot \nu
$$

where $t_{\text {system }}$ is chosen equal to $0.5 \mathrm{~s}$, if the target vehicle has CACC, and $1.4 \mathrm{~s}$ otherwise.

The constant factor $k$ was chosen equal to 0.3 in accordance with earlier MIXIC studies [12]. The constant factor $k_{a}$ was chosen equal to 1.0 in accordance with [6]. The default values of MIXIC for $k_{\nu}$ and $k_{d}$ are 3.0 and 0.2 , respectively. In a recent MIXIC study, these values were set more "strongly" to 0.58 and 0.1 , respectively [17]. Fig. 2 illustrates how combinations of the respective CACC parameters of the reference-acceleration function (by systematically varying the values for $k_{\nu}$ and $k_{d}$ )

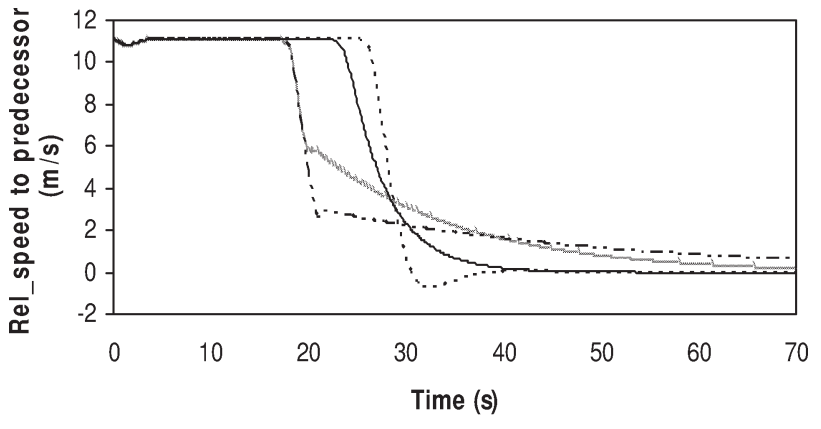

$\mathrm{Kd}=0.2 ; \mathrm{Kv}=3.0 ; \mathrm{Ka}=1.0-\cdots \cdot \mathrm{Kd}=0.1 ; \mathrm{Kv}=3.0 ; \mathrm{Ka}=1.0$
$\cdots \cdots \cdot \mathrm{Kd}=0.2 ; \mathrm{Kv}=0.58 ; \mathrm{Ka}=1.0-\mathrm{Kd}=0.1 ; \mathrm{Kv}=0.58 ; \mathrm{Ka}=1.0$

Fig. 2. Relative speed of a CACC vehicle approaching a slower CACC equipped vehicle.

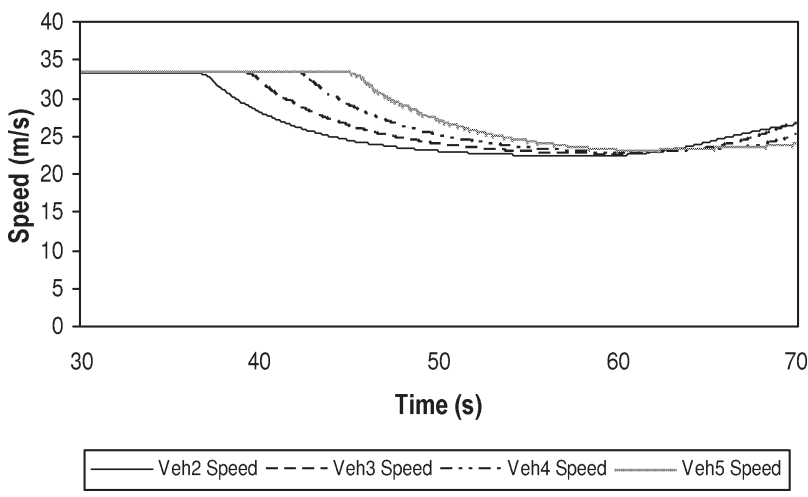

Fig. 3. Speed of manually controlled vehicles.

influence the relative speed of a CACC-equipped vehicle approaching a slower CACC-equipped vehicle.

In this paper, the parameter setting of $k_{d}=0.1, k_{\nu}=0.58$, and $k_{a}=1.0$ was selected, since this setting resulted in the most smooth and fast reaction of the CACC controller without leading to unsafe situations compared to the other settings (Fig. 2).

Regarding the human interaction with the CACC, it is assumed (similarly to the operation of an ACC in MIXIC) that the driver can switch the system on and off. The driver will switch on the CACC as much as possible but will turn the CACC off if a deceleration required is stronger than the CACC capability or in case of a mandatory lane change.

Further tests to check the CACC operation in MIXIC were performed, assuming a platoon of five vehicles. The first vehicle drives at $80 \mathrm{~km} / \mathrm{h}$, and the other vehicles approach in a platoon of four vehicles. In the reference situation, all vehicles are under manual control; in the other situation, the vehicles are CACC controlled. Fig. 3 displays the speed of manually controlled vehicles. Fig. 4 displays the speed of the CACC-controlled vehicles.

We conclude from Figs. 3 and 4 that the approaching CACCequipped vehicles react faster on the decelerating predecessor than in the scenario in which all vehicles are manually driven. The lines in the diagrams of the $100 \%$ CACC-equipped platoon stick close together, which indicates that the time between an accelerating or decelerating action of the predecessor and the 


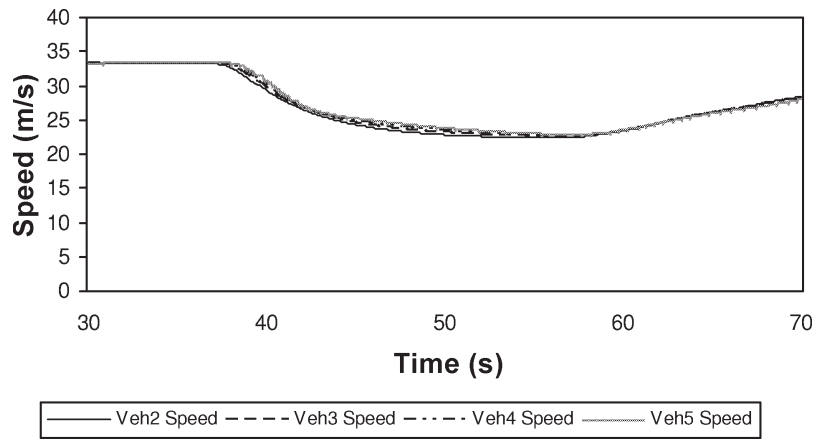

Fig. 4. Speed of CACC controlled vehicles.

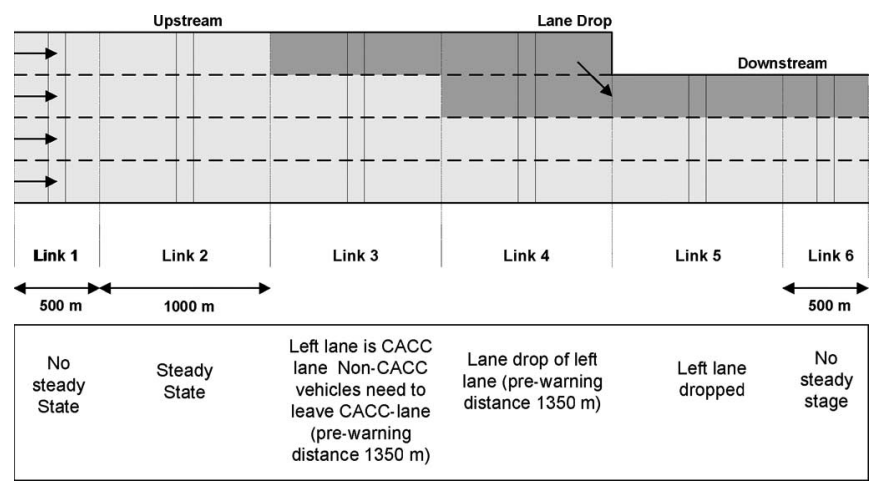

Fig. 5. Simulated highway configuration.

same action of the successor is less than in the scenario of $100 \%$ manually driven vehicles. Furthermore, the curves of the CACC-equipped platoon are smoother, which illustrates a smoother behavior of the CACC-equipped vehicles above manually driven vehicles. Analysis of the acceleration reveals that the CACC vehicles do not decelerate stronger than $-2 \mathrm{~m} / \mathrm{s}^{2}$, while the manually driven vehicles decelerate as strong as $-2.5 \mathrm{~m} / \mathrm{s}^{2}$.

\section{Simulation Setup}

The traffic simulation model MIXIC was used to examine the impact of CACC on the traffic flow. The basic configuration used in this paper is a four-lane highway with a road narrowing by a lane drop. A lane drop corresponds to a mandatory lane change in the MIXIC traffic simulation model. When a mandatory lane change is carried out, the drivers turn off their CACC system. Once the mandatory lane change has been carried out, the system is turned back ON under the normal conditions maintained by MIXIC. A lane drop makes it possible to measure the maximum traffic volume at different penetration rates of CACC when the traffic volume on the link before the lane drop nears a congestion state. In addition, a number of experiments were conducted, with a special lane for CACC vehicles, to study whether this would lead to additional trafficflow benefits. The highway configuration, presented in Fig. 5, is split into six links of $1000 \mathrm{~m}$ each. A left lane drop is modeled from $4 \mathrm{~km}$ after the start of the simulation.

A prewarning of the "merge" is given to the driver, $1350 \mathrm{~m}$ before the transition, from four to three lanes. In the scenarios with a CACC lane, the CACC lane is introduced after $2000 \mathrm{~m}$ on the left most lane, expanded with one lane after $3000 \mathrm{~m}$, after which the left most CACC lane ends after $4000 \mathrm{~m}$ (see dark lane sections). CACC drivers were not assumed to go to the CACC lane consciously. However, when they are driving on the CACC lane, they will not leave it. The simulation time was $150 \mathrm{~min}$ per run. A measurement point is placed in the middle of each link for statistical analysis. Data from the first and last sections were not analyzed to avoid transient aspects.

For the traffic generation, data from the A4 highway, near Schiphol in The Netherlands, was used. The MIXIC behavior for this data set was previously calibrated [12]. The data set contains sufficiently high-traffic volumes (up to $7600 \mathrm{pcu} / \mathrm{h}$ ) that can lead to congestion in the narrowing scenario under study. The number of trucks and vans in this data set is small (vehicles $94 \%$, vans $4 \%$, and trucks $2 \%$ ).

Regarding the operation of the CACC, the time-gap setting of the CACC system is set on $0.5 \mathrm{~s}$, if it is following a CACCequipped vehicle, and on $1.4 \mathrm{~s}$, if it is following a non-CACCequipped vehicle, respectively. The penetration rate of CACC systems was varied in multiples of $20 \%$. This resulted in one reference case with no CACC vehicles, five CACC scenarios without a CACC lane, and three CACC scenarios with a CACC lane.

To ensure statistical validity, five stochastically independent simulations were performed for each selected scenario. Analysis of variance (ANOVA) was used to test whether the means of an indicator in different scenarios were significantly different from each other. Post hoc Tukey's tests were used to study whether the value of an indicator on a specific link differed from the values on other links. To test to what extent a CACC scenario was significantly different from the reference case, post hoc Dunett's tests were performed. These tests enable to compare the means of a group to a control group. In this case, the control group is the 0\%-CACC scenario (reference case).

\section{Simulation Results}

We illustrate the results by the following output variables that were analyzed on link four (just before the lane drop) and link five (just after the lane drop). Since shockwaves represent variations in the flow that propagate through the traffic, the number of shock waves on a highway stretch is used as a measure for traffic stability. It is defined as an observation of at least three vehicles on the same lane within a mutual distance of $50 \mathrm{~m}$ and within a time period of $3 \mathrm{~s}$ with a deceleration stronger than $-5 \mathrm{~m} / \mathrm{s}^{2}$. Furthermore, we used the average speed on a link as an indicator for traffic throughput. Finally, we measured, the three highest 5-min average traffic volumes are used as an indication of the roadway capacity.

In general, we observed that during the simulations, a number of vehicles did not succeed to merge from link four to link five (i.e., these vehicles were removed from the simulation). This especially occurred in the case of a CACC lane and in the case of a high-penetration of CACC vehicles, leading to reduced merging gaps because of the close following.

Fig. 6 shows that the number of shockwaves, just before the lane drop, decreases drastically when more CACC-equipped vehicles are present. The same was found just after the lane 


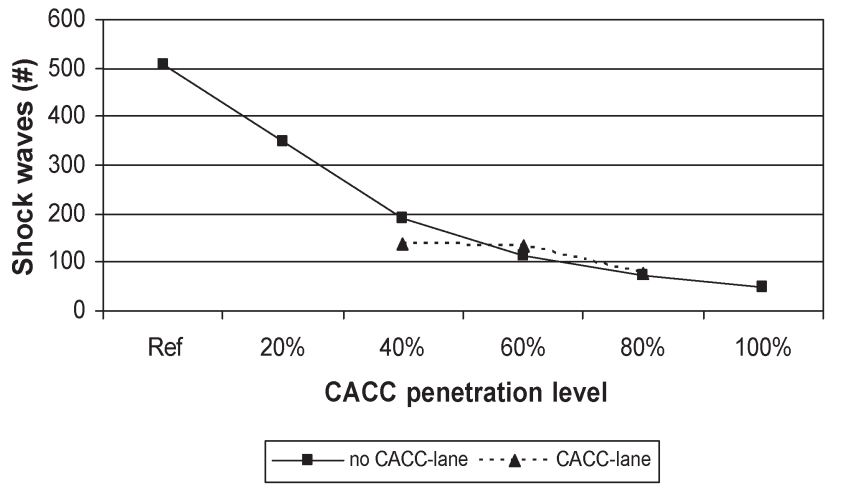

Fig. 6. Number of shockwaves just before the lane drop (link four).

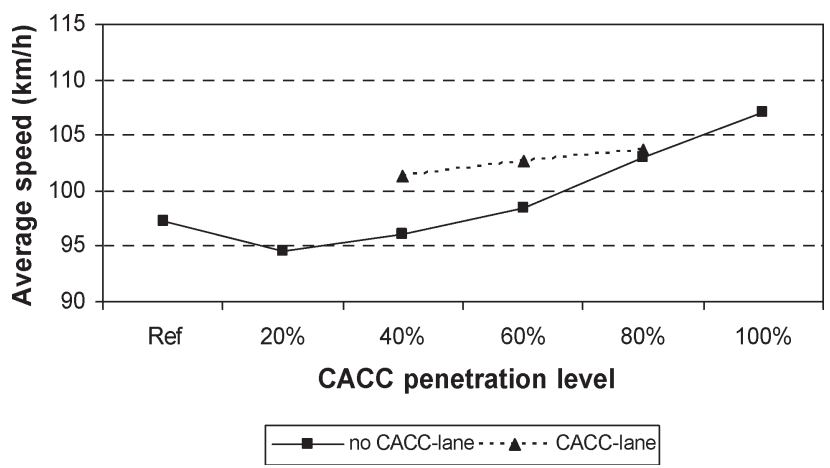

Fig. 7. Average speed just before the lane drop (link four).

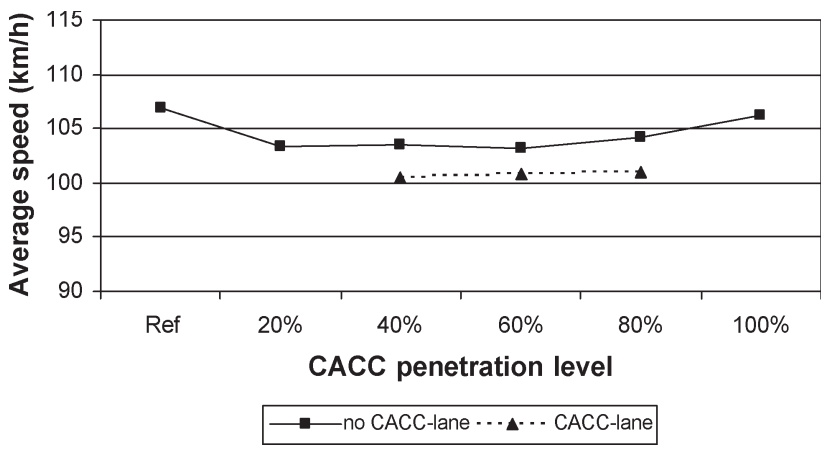

Fig. 8. Average speed just after the lane drop (link five).

drop, although the absolute numbers were much lower. This reduction is significant for all CACC cases and especially in comparison with the reference case. The scenarios in which a regular lane is replaced by a CACC lane report the same patterns as the CACC scenarios without a CACC lane. Additionally, it should be noticed that the characteristics of a shock wave, in terms of average number of vehicles in a shockwave, number of observations, and speed of shock waves, do not change significantly for the CACC scenarios analyzed.

The average speed observations on the different links show a different pattern for the scenario with and without CACC lane. We show the results for link four (Fig. 7) and link five (Fig. 8).

For the scenarios without CACC lane, the results show that the average speed decreases with respect to the reference scenario if small fractions of CACC-equipped vehicles are introduced. Introducing more CACC-equipped vehicles results in a recovery of the average speed. Remarkably, Fig. 7 shows

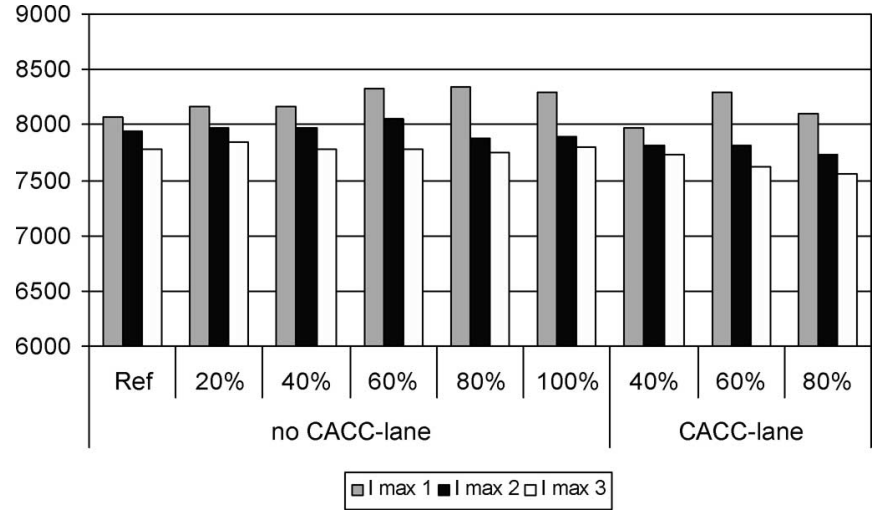

Fig. 9. Potential impact on highway capacity just after the lane drop (link five).

that the average speed is higher if there is a CACC lane. This can be explained by the fact that because of the CACC lane, there are many more lane changes required for both normal and CACC-equipped vehicles and that most of these lane changes are also performed earlier on link two and link three. This explanation is confirmed by the fact that on link three, the average speed for the scenarios with CACC lane was indeed much lower. Therefore, recovery of the average speed starts earlier. However, the recovery speed is lower for the scenarios with CACC lane (Fig. 8). This difference is caused by the lower average speed on the non-CACC lanes.

To study the potential impact of CACC on highway capacity, Fig. 9 gives the three highest 5-min average traffic volumes ( $\mathrm{pcu} / \mathrm{h}$ ) measured on link five, just after the lane drop.

The maximum observed traffic volumes on the link before the bottleneck show only small differences for different CACCpenetration levels. For the link just after the lane drop, ANOVA shows a significant impact of CACC on the highest maximum traffic volume if no CACC lane is present for $60 \%$ and $80 \%$ penetration. However, on the second and third highest maximum traffic volume, no significant effect of CACC is established. The replacement of a regular lane for a CACC lane does not improve the maximum traffic volume of the link after the bottleneck. Even a slight degradation of performance can be observed, with respect to similar CACC fractions, when no CACC lane is present.

\section{DISCUSSION}

In this paper, it is concluded that CACC is able to improve traffic-flow characteristics. The expectation that a lowpenetration rate of CACC $(<40 \%)$ does not have an effect on traffic flow throughput is correct. A reduction in the number of shock waves is demonstrated on the links with relatively hightraffic volumes (links four and five). However, this does not significantly affect traffic throughput in terms of higher speeds. Even a small decrease in average speed is established when only a small number of vehicles are CACC equipped.

The expectation that a high-CACC-penetration rate $(>60 \%)$ has benefits on traffic stability and throughput appears to be right. However, the level of improvement does heavily depend on the traffic-flow condition. During high-traffic volume, there is more interaction between vehicles than during low-traffic 
volume. As a result, more vehicles are able to participate in a CACC platoon. Since CACC reduces time gaps and improves string stability, traffic flow especially improves in conditions with high-traffic volume. This is indicated by a higher average speed and a high reduction of the number of shock waves and standard deviation of speed on the link before the bottleneck.

The presence of a special CACC lane in combination with low-CACC fractions leads to a degradation of performance. The $20 \%$-CACC scenario even results in severe congestion on the link before the lane drop. The expectation that in the presence of a CACC lane, higher CACC fractions could lead to an improvement of traffic flow on the highway with respect to the same CACC-penetration rate, and no CACC lane is only confirmed by the MIXIC simulations for the high-volume stretch before the bottleneck. A CACC lane improves traffic flow on link four with respect to the scenarios without CACC lane as well as the reference case. This is demonstrated by higher speeds and lower standard deviations of speed. An explanation for this good performance of the CACC lane is the division over the lanes. On link four, it is shown that halfway of link four, all mandatory lane changes performed by nonequipped vehicles are performed, which indicates that traffic is in a steady state sooner after the merging process.

If communication between vehicles is restricted to longitudinal control, the system has a negative effect on traffic safety in the merging process. Close-CACC platoons prevent other vehicles from merging, because the gap between consecutive CACC vehicles is smaller than the gap accepted for performing a mandatory lane change. The unsafe lateral effect of the presence of $\mathrm{CACC}$ in traffic is revealed by an increasing number of lane-change failures as more CACC-equipped vehicles are present in traffic. A few options for the improvement of the CACC system, on this paper, may be considered. If vehicles want to merge into a CACC platoon but cannot find a proper gap, a gap should be created, either on instigation of the merging vehicle or a roadside beacon. In addition to that, a cooperative-merging procedure could be designed, allowing a CACC vehicle to smoothly merge with other CACC vehicles, without introducing new disturbances. Finally, the length of a platoon with closely driving-CACC vehicles could be limited, enabling a merging vehicle to find an appropriate gap more easily.

Regarding the potential positive effects of CACC on highway capacity, there are some indications that capacity increases after the lane drop. However, this could not be established explicitly by the MIXIC simulations. This may be explained by the fact that the traffic volumes on the link, after the lane drop in the reference case, are rather high $(8000 \mathrm{pcu} / \mathrm{h})$. This may appear high when compared with, e.g., the capacity estimate of $7250 \mathrm{pcu} / \mathrm{h}$ given by Dutch highway-capacity manual [18]. However, the $7250 \mathrm{pcu} / \mathrm{h}$ estimate is based on a $15-\mathrm{min}$ measurement interval, while the $8000 \mathrm{pcu} / \mathrm{h}$ is based on a 5 -min measurement interval, and indeed, these traffic volumes are regularly observed on Dutch highways. Reflecting on this, there are not much possibilities for an enhancement of highway capacity. Given this ceiling effect, gaining traffic-flow stability at these high levels of throughput must be considered of great value.

\section{CONCLUSION}

This traffic simulation study examined to what extent CACC can contribute to a better traffic-flow performance. CACC is a communication-based system, which is designed to enhance driving comfort on highways by relieving the driver of the need to continually adjust his speed to match that of preceding vehicles while also maintaining constant time gap. A literature survey to both the functionality and potential traffic-flow effects indicates that although CACC is designed as a comfort system, it is supposed that the system has an impact on traffic flow when widely recognized and used. However, not much research has been done regarding to the traffic-flow impacts of CACC. The main contributions of this paper are that CACC, indeed, shows potential positive effects on traffic throughput; furthermore, CACC reveals to increase highway capacity near a lane drop.

The microscopic-traffic simulation model MIXIC was used to examine the impact of CACC on the traffic flow. The functionality of CACC is elaborated in functional specifications for MIXIC, and the model is extended with this functionality.

MIXIC simulations with data measured on a four-lane Dutch highway, with a bottleneck due to a lane drop, indicate that CACC has the ability to improve traffic-flow performance. However, to what extent depends heavily on the traffic-flow conditions on the highway stretch and the CACC-penetration rate. The traffic flow especially improves in conditions with high-traffic volume and when high fractions of the vehicle fleet are CACC equipped. Then, more vehicles are able to participate in a CACC platoon, resulting in reduced time gaps and improved string stability.

The impact of a dedicated lane for CACC-equipped vehicles depends heavily on the CACC-penetration rate. A low-CACC presence $(<40 \%)$ leads to a degradation of performance, which is demonstrated by lower speeds, higher speed variances, and more shock waves. Furthermore, the CACC lane improves traffic performance, but only for the high-volume stretch before the bottleneck. This is demonstrated by higher speed and lower speed variances.

The traffic volumes on the link after the lane drop are relatively high compared to generally accepted capacity estimates. Nonetheless, the presence of CACC in this traffic situation shows an enhancement of highway capacity. Such capacity enhancement, during already high-traffic volume, can be regarded as an important finding.

As communication is restricted to longitudinal control and no restrictions to the length and compactness of CACC platoons is given, the system has a negative effect on traffic safety in the merging process. Close-CACC platoons prevent other vehicles from cutting in, resulting in an increasing number of removed vehicles due to conflicts, as more vehicles are CACC equipped. It is recommended to study possible solutions for dealing with this negative effect of CACC on the merging process. Options for improvement are 1) limiting the length of a CACC platoon; 2) an infrastructural beacon on the highway stretch before a bottleneck, communicating to vehicles that they should enlarge the time gap to its predecessor enabling vehicles to perform a (mandatory) lane change; and 3) adding a 
"cooperative-merging" application to the CACC system, enabling vehicles to communicate their lateral (lane change) movements.

The results in this paper have contributed to the understanding of the impacts of CACC on traffic flows. To a certain extent, they have busted the myth that CACC can strongly increase roadway capacity. Nevertheless, some open issues are to be addressed. First, traffic simulations are notoriously weak in modeling congestion. It is recommended to specifically develop realistic congested simulation scenarios and assess the impact of CACC in these situations. Second, there may be an interaction between traffic-flow effects and communication and sensor-system characteristics and limitations; these aspects have not been addressed so far.

\section{REFERENCES}

[1] Ministry of Transport, Public Works and Water Management, Nota Mobiliteit; Naar een betrouwbare en voorspelbare bereikbaarheid, 2004, The Hague. (in Dutch).

[2] European Commission, Final Report of the eSafety Working Group on Road Safety, Nov. 2002, Brussels, Belgium: EC DG IST.

[3] P. J. Zwaneveld and B. van Arem, "Traffic effects of automated vehicle guidance system. A literature survey," TNO Inro, Delft, The Netherlands, Report INRO-VVG 1997-17, 1997.

[4] A. E. Hoetink, "Advanced Cruise Control en verkeersveiligheid," Inst. for Road Safety Res. (SWOV), Leidschendam, The Netherlands, R-2003-24, 2003. (in Dutch).

[5] B. van Arem, J. H. Hogema, M. J. W. A. Vanderschuren, and C. H. Verheul, "An assessment of the impact of autonomous intelligent cruise control," TNO Inro, Delft, The Netherlands, INRO-VVG 1995-17a, 1995.

[6] J. VanderWerf, S. E. Shladover, M. A. Miller, and N. Kourjanskaia, "Evaluation of the effects of adaptive cruise control systems on highway traffic flow capacity and implications for deployment of future automated systems," Transp. Res. Rec. 1800, pp. 78-84, 2003.

[7] Automated Highway Systems, P. A. Ioannou, Ed. New York: Plenum, 1997.

[8] B. van Arem, C. M. J. Tampère, and K. M. Malone, "Modeling traffic flows with intelligent cars and intelligent roads," in Proc. IEEE Intell. Vehicles Symp., Columbus, OH, Jun. 2003, pp. 456-461.

[9] K. M. Malone and B. van Arem, "Traffic effects of inter-vehicle communication applications in CarTALK 2000," presented at the 11th World Congr. Intell. Transp. Syst., Nagoya, Japan, Oct. 2004, Paper 2156.

[10] T. Benz, T. Dieckmann, G. Fisanotti, T. Geißler, B. Harker, R. Herrmann, C. Kanz, G. Künzel, C. Lanfranco, S. Martini, V. Murdocco, M. Bellezza, R. Montanari, E. Poyet, V. Puglia, W. Schulz, M. Schulze, and A. Stenman, "CHAUFFEUR 2: Final Rep. Deliverable D24," DaimlerChrysler, Stuttgart, Germany, 2003.

[11] B. van Arem, A. P. de Vos, and M. J. W. A. Vanderschuren, "The microscopic traffic simulation model MIXIC 1.3," TNO Inro, Delft, The Netherlands, INRO-VVG 1997-02b, 1997.

[12] — - "The Effect of a Special Lane for Intelligent Vehicles on Traffic Flows," TNO Inro, Delft, The Netherlands, INRO-VVG 1997-02a, 1997.

[13] G. O. Burnham, J. Seo, and G. A. Bekey, "Identification of human driver models in car following," IEEE Trans. Autom. Control, vol. AC-19, no. 6, pp. 911-915, Dec. 1974.

[14] B. van Arem, A. P. de Vos, and H. Schuurman, "Simulation of traffic flow on a special lane for intelligent vehicles," in Proc. 3rd Int. TRB Symp. Highway Capacity, Copenhagen, Denmark, Jun. 1998, pp. 71-83.

[15] J. A. C. van Toorenburg, Praktijkwaarden voor de capaciteit. Rotterdam, The Netherlands: Transp. Res. Centre (DKV), Ministry Transp. Public Works and Water Manage., 1986 (in Dutch).

[16] J. Vanderwerf, N. Kourjanskaia, S. E. Shladover, M. Miller, and H. Krishnan, "Modeling the effects of driver control assistance systems on traffic," Transp. Res. Rec. 1748, pp. 167-174, 2001.

[17] K. M. Malone, "CarTALK," TNO Inro, Delft, The Netherlands, Final Rep. 04-7N-141-72541, 2004.

[18] AVV Transport Research Centre, Handboek Capaciteitswaarden Infrastructuur Autosnelwegen, 1999, Rotterdam, The Netherlands: Ministry Transp. Public Works and Water Manage. (in Dutch).

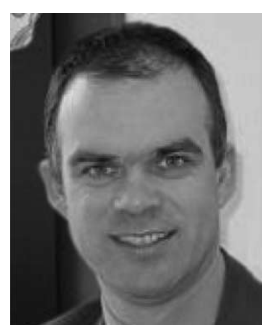

Bart van Arem (M'04) received the Master's and the $\mathrm{Ph} . \mathrm{D}$. degrees in applied mathematics, specialty on queuing theory, from the University of Twente, Enschede, The Netherlands, in 1986 and 1990, respectively.

In 1991, he was engaged in modeling traffic flows at roundabouts for the Dutch Ministry of Transport Public Works and Water Management. Since 1992, he has been working as a Researcher at The Netherlands Organization for Applied Scientific Research TNO on numerous intelligent-transport systems (ITS) related projects. Since 2003, he has been a Professor with the Applications of Integrated Driver Assistance (AIDA) at the University of Twente, and is currently leading the knowledge center AIDA. His current interest focuses on advanced driver-assistance systems, specializing on impact assessment, scenario development, and traffic-flow modeling.

Dr. van Arem is Editor-In-Chief of the IEEE Intelligent Transportation Systems Society Newsletter. Furthermore, he is a member of the eSafety WG RTD and the International Task-Force Vehicle-Highway Automation.

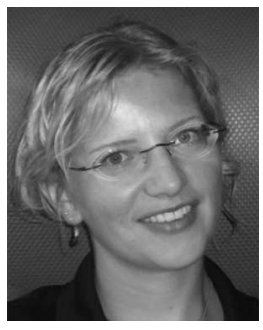

Cornelie J. G. van Driel received the M.Sc. degree in civil engineering and management from the Centre for Transport Studies, University of Twente, Enschede, The Netherlands, in 2001. Since 2003, she has been working toward the Ph.D. degree in civil engineering and management. Her Ph.D. research is on the assessment of integrated driver assistance at the knowledge center AIDA.

From 2001 to 2003, she has worked as a Researcher at the Centre for Transport Studies. Her research interests are in the areas of intelligent-transport systems, advanced driver-assistance systems, user needs, human-machine interaction, traffic safety, and traffic efficiency.

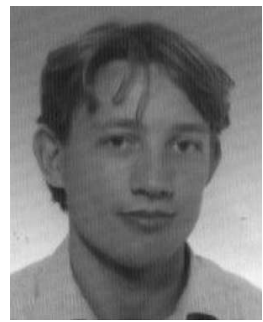

Ruben Visser received the M.Sc. degree in civil engineering and management from the Centre for Transport Studies, University of Twente, Enschede, The Netherlands, in 2005.

In July of 2005, he started working at 4Motion Consultancy B.V., Utrecht, The Netherlands, a company that assists governmental organizations in realizing projects in the area of traffic and transportation, infrastructure, and spatial development. 